\title{
Dynamic balance in children with attention-deficit hyperactivity disorder and its relationship with cognitive functions and cerebellum
}

This article was published in the following Dove Press journal:

Neuropsychiatric Disease and Treatment

2I March 2017

Number of times this article has been viewed

\author{
Michal Goetz' \\ Jaroslava Paulasova \\ Schwabova ${ }^{2}$ \\ Zdenek Hlavka ${ }^{3}$ \\ Radek Ptacek ${ }^{4}$ \\ Craig BH Surman ${ }^{5}$ \\ 'Department of Child Psychiatry, \\ Second Faculty of Medicine, Motol \\ University Hospital, ${ }^{2}$ Department of \\ Neurology, ${ }^{3}$ Department of Statistics, \\ ${ }^{4}$ Department of Psychiatry, Charles \\ University, Prague, Czech Republic; \\ ${ }^{5}$ Department of Psychiatry, Harvard \\ Medical School, Boston, MA, USA
}

Background: Attention-deficit hyperactivity disorder (ADHD) is linked to the presence of motor deficiencies, including balance deficits. The cerebellum serves as an integrative structure for balance control and is also involved in cognition, including timing and anticipatory regulation. Cerebellar development may be delayed in children and adolescents with ADHD, and inconsistent reaction time is commonly seen in ADHD. We hypothesized that dynamic balance deficits would be present in children with ADHD and they would correlate with attention and cerebellar functions.

Methods: Sixty-two children with ADHD and no other neurological conditions and 62 typically developing (TD) children were examined with five trials of the Phyaction Balance Board, an electronic balancing platform. Cerebellar clinical symptoms were evaluated using an international ataxia rating scale. Conners' Continuous Performance Test was used to evaluate patterns of reaction.

Results: Children with ADHD had poorer performance on balancing tasks, compared to TD children $(P<0.001)$. They exhibited significantly greater sway amplitudes than TD children $(P<0.001)$ in all of the five balancing trials. The effect size of the difference between the groups increased continuously from the first to the last trial. Balance score in both groups was related to the variation in the reaction time, including reaction time standard error $(r=0.25 ; P=0.0409$, respectively, $r=0.31 ; P=0.0131)$ and Variability of Standard Error $(r=0.28 ; P=0.0252$, respectively, $r=0.41 ; P<0.001)$. The burden of cerebellar symptoms was strongly related to balance performance in both groups ( $r=0.50, P<0.001 ; r=0.49, P=0.001)$.

Conclusion: This study showed that ADHD may be associated with poor dynamic balance control. Furthermore, we showed that maintaining balance correlates with neuropsychological measures of consistency of reaction time. Balance deficits and impaired cognitive functioning could reflect a common cerebellar dysfunction in ADHD children.

Keywords: attention-deficit hyperactivity disorder, balance, cerebellum, reaction time

\section{Introduction}

Attention-deficit hyperactivity disorder (ADHD) is a frequent, highly heritable neurodevelopmental condition characterized by an age-inappropriate level of inattention (IA) and hyperactivity-impulsivity (HI). Besides a number of psychiatric comorbidities, ADHD is linked to the frequent presence of motor deficiencies, including fine as well as gross motor skills. ${ }^{1-7}$ Several studies have identified balance deficits in children and adults with ADHD. ${ }^{6-12}$ The maintenance of balance in static and dynamic conditions represents a crucial motor function and is required for the development and execution of a wide variety of more complex motor skills.
Correspondence: Michal Goetz Department of Child Psychiatry, Second Faculty of Medicine, Motol University Hospital, Charles University, V Uvalu 84, 15006 Prague 5, Czech Republic Tel +420 7 287I 8595

Email michal.goetz@lfmotol.cuni.cz 
Balance, especially its dynamic part, is important in ordinary life because it enables activities to be performed while moving, and any malfunction increases the risk of injury. This is indeed relevant in ADHD, which is associated with elevated risk for a range of injuries. ${ }^{13,14}$ Surprisingly, only a few studies have studied dynamic balance in individuals with ADHD. ${ }^{6,11,15}$

Buderath et $\mathrm{al}^{6}$ investigated balance abilities in children with ADHD using static and dynamic posturography. Balance problems were the most prominent in the dynamic task. ${ }^{6}$ Mao et $\mathrm{al}^{11}$ demonstrated that children with ADHD had less effective balance strategies than a control group when riding a mechanical horse. Finally, Ren et al, ${ }^{15}$ using computerized dynamic posturography, found that ADHD boys had poorer postural control ability and impaired visual and vestibular information processing in comparison with typically developing (TD) children.

The management of balance is mediated by the coordinated function of the visual, vestibular, proprioceptive, musculoskeletal, and central nervous systems. ${ }^{16}$ The cerebellum plays an integrative role in the management of balance, particularly the vermis and paravermal area. In parallel, volumetric abnormalities of the cerebellar vermis are among the most frequent findings in structural studies of ADHD. ${ }^{17-22}$ Balance problems in children with ADHD were similar to those found in children with vermal or fastigial nucleus lesion. ${ }^{6}$ Besides the role in balance and other motor functions, the cerebellum is involved in the regulation of attention and other cognitive functions. ${ }^{23}$ Cerebellar activation has been noted during Continuous Performance Tests (CPTs), which require attention processes, in functional magnetic resonance imaging (fMRI) investigations. ${ }^{24,25}$

We sought to confirm that balance deficits are present in children with ADHD and that these balance deficits correlate with control over reaction and correlate with symptoms of cerebellar dysfunction. To these ends, we conducted the first study of electronic balancing board and CPT task performance in children with ADHD. We chose to use an electronic balancing board, which has not been used in previous studies of ADHD, to allow sensitive measure of balancing abilities.

\section{Methods}

\section{Sample}

The sample was recruited via the outpatient services of the Department of Child and Adolescent Psychiatry at Motol University Hospital in Prague and from a local hospital outside of the capital. A total of 104 Caucasian children attending regular basic school aged 7-11 years who were consecutively referred to the clinics due to suspected ADHD were invited to participate in the study. The psychiatric evaluation consisted of a semistructured clinical interview for mental disorders that was developed at Motol University Hospital based on institutional consensus. A mother of each participant completed the parent version of the screening questionnaire for symptoms of mental disorders, the Child Symptom Inventory-4 (CSI-4). ${ }^{26}$ The CSI-4 ADHD subscale consists of 18 questions on all symptoms of ADHD. The severity of each symptom is classified using a Likert scale (0 - never, 1 - sometimes, 2 - often, and 3 - very often). The CSI-4 scale, including its Czech version, was used in several multicenter international studies on ADHD. ${ }^{27,28}$ Teachers completed a school-oriented questionnaire designed to assess academic, behavioral, and social difficulties. We required that both parents and teachers described problems with attention and/or problems with hyperactivity and impulse control in order to suspect a diagnosis of ADHD. Participants also underwent a neurological examination by a pediatric neurologist.

Based on structured clinical interview and questionnaires, 90 children fulfilled the Diagnostic and Statistical Manual of Mental Disorders, Fourth Edition (DSM-IV; American Psychological Association, 199429) criteria for ADHD. The exclusion criteria were as follows: tic disorder and other neurological illnesses that might influence the maintenance of balance or participation in the study or be likely to reflect possible cerebellar dysfunction such as known cognitive impairment, learning disabilities, or pervasive spectrum disorders. Finally, children treated with atomoxetine or other medications for behavioral problems, with the exception of methylphenidate, were excluded due to an unacceptably long washout period. Finally, children with serious symptoms of physical aggression when untreated were excluded. Ultimately, 62 children with a DSM-IV diagnosis of ADHD (55 boys and 7 girls: age $7-11$ years, $M$ age $=8.79$ years, standard deviation $[\mathrm{SD}]=1.32$ ) were enrolled in the study. These children presented with a combined subtype $(90.3 \%$, $n=56)$, inattentive subtype $(6 \%, n=4)$, or a hyperactiveimpulsive subtype $(3 \%, \mathrm{n}=2)$. Comorbidities included oppositional defiant disorder (ODD) in 35 children $(56 \%)$ and anxiety disorders in 4 children (6\%). The majority of the children (95\%) were medication-naive. Patients who were being treated using methylphenidate (children were treated for only a few weeks before entering the study) participated in a 48- to 72-hour washout period prior to testing. We considered the washout period long enough that these 
children's performance would be comparable with the rest of the sample.

\section{Controls}

A sample of 62 sex- and age-matched ( \pm 6 months) TD schoolchildren ( 58 boys and 7 girls: age $=8.76$ years, $\mathrm{SD}=1.36$ ) was recruited from elementary schools near the study center. Teachers were asked to select children without learning disabilities, attention problems, or signs of hyperactivity or impulsivity. Teachers then delivered an invitation letter to parents requesting that their children be allowed to participate in the study. The parents of these children confirmed that they had not observed any evidence of learning disabilities, attention problems, hyperactive behaviors, impulsivity, or neurological illnesses in their children and completed the parent version of the CSI-4. The same child psychiatrist who evaluated the clinical sample reviewed all of the children's records and interviewed the TD children to confirm the absence of ADHD.

\section{Balance examination}

Studies show that balance deficits manifest when vision is disrupted ${ }^{6,30}$ or when the test is dynamic. ${ }^{11}$ The investigations of balance abilities were performed using the unstable electronic Phyaction Balancing Board (GymnaUniphy, Bilzen, Belgium). This $10 \mathrm{~cm}$ high board has a wide antislip area of $42 \times 42 \mathrm{~cm}^{2}$ and allows balance in a side-to-side (lateral) direction, requiring the participant to resist tipping to the left and right sides to keep the board parallel to the floor. Any deviation in the surface is converted into an electrical signal that is then on the sum of the area defined by the path of a point oscillating from the imaginary center and a line bisecting the center. The subject is required to stand on a set location on the surface, hold his hands by his sides, and look forward. The investigator holds the balance board so that it does not move, and the subject can assume the balanced position. Once the subject is in position and indicates that he or she is ready, the investigator lets go of the balance board. The subject then tries to maintain the surface in a central, ie, balanced, position. An investigator stood nearby to provide support should the subject fall off the balance board. Each patient performed five trials in the lateral (side-to-side) direction, which lasted a total of 3.5 minutes.

\section{Examination of cerebellar symptoms}

The children with ADHD and the TD children were examined using the International Cooperative Ataxia Rating Scale (ICARS). ${ }^{31}$ The evaluation was performed by a board-certified child neurologist. The ICARS is a 100-point scale that is divided into the following sections: postural and stance disturbances, limb movement disturbances, speech problems, and oculomotor dysfunction. The reliability and validity of the ICARS have been confirmed by several studies on individuals suffering serious cerebellar pathology; ${ }^{32,33}$ furthermore, the ICARS has previously been used in a study of handwriting in children with ADHD. ${ }^{34}$

The evaluator was not blind to the group status of the children but was blind to the balance performance of evaluated subjects.

\section{Neuropsychological testing}

All the participants completed the Conners' CPT-II ${ }^{35}$ on the same laptop. All the participants underwent testing between 9 am and $12 \mathrm{pm}$.

\section{Statistical analysis}

The sample size was selected according to a power analysis with the intention of revealing moderate Spearman's correlations with $r$ coefficients $=0.4$, an alpha error $=0.05$, and a beta error $=0.1$ using the statistical software MedCalc for Windows Version 10.0.1.0 (MedCalc Software, Ostend, Belgium). Group differences were analyzed using standard two-sample $t$-tests with Welch approximations of the degrees of freedom. The effect size was estimated using Cohen's $d$ and Pearson's $r$ coefficients.

To reduce the distorting effects of outlier balance measures, we used a Box-Cox power transformation with a coefficient of $1 / 3$ (cube root transformation). Principal components analysis revealed that $78.52 \%$ of the total variability of cube roots of the five balance measurements is explained by the first principal component corresponding to a sample mean of the measurements. Therefore, we define a measure of overall score (MeanBal) as the arithmetic average of the cube roots of the five measurements. We found that the second principal component explained an additional 11.61\% of total variability, which can be interpreted as a measure of improvement over the five trials.

The strengths of the unadjusted relationships between ADHD symptom scores, variables from CPT-II, and the balance measures were evaluated by calculating Spearman's rank correlation coefficients $(\rho)$ separately for the children with ADHD and the TD participants.

The effect of the overall balance measure (MeanBal) on the age-corrected ADHD symptom scores as well as variables from CPT-II was evaluated using linear regression. First, the regression analysis with correction for age was performed 
separately for the children with ADHD and the TD children. Then the analysis was performed in the whole sample with the effects of the balance measure in this model corrected for age and group (ie, ADHD/TD). We also tested whether the regression coefficients that measured the strengths of the relationships between the overall balance score (MeanBal) and the behavioral markers were similar in both groups by testing the interaction between the group (ADHD/TD) and the balance score (MeanBal). The analyses were performed using the statistical computing environment R 3.0.0 (R Core Team, 2013). ${ }^{36}$

\section{Ethics}

This study was approved by the institutional review board of the Motol University Hospital in Prague. A written informed consent was obtained from the parents of the children with ADHD and the control subjects. The children provided a written assent for the study.

\section{Results}

\section{Group differences in balance and CPT}

All participants completed the study. We found that the children with ADHD had poorer performance at the balancing tasks, reflected in higher values of overall balance score (MeanBal) compared to TD children $(P<0.001)$. Children with ADHD exhibited significantly greater swaying amplitudes than TD children $(P<0.001)$ in all of the five balancing trials (Figure 1). Several falls from the balance board occurred in the ADHD group but not in the TD group. The effect size of the difference between the groups increased continuously from the first to the last trial (Table 1), suggesting worse improvement in performance in the ADHD group. The ADHD group differed from the TD group on all CPT-II variables except for commission errors, detectability $(\delta)$, and Hit-SE-Block (Table 1).

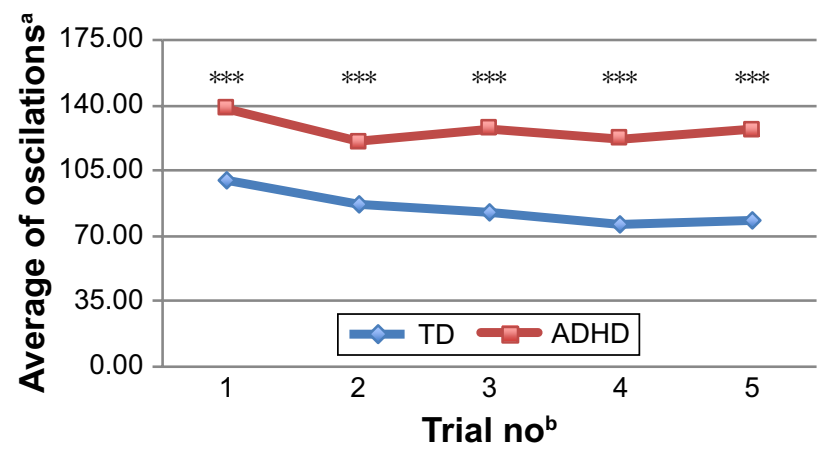

Figure I Laterolateral balance measures.

Notes: ${ }^{\text {TT }}$ The sum of the area, which is defined by the path of a point oscillating from the imaginary center and a line bisecting the center. Higher number means worse performance. ${ }^{\mathrm{b}}$ Trial on the balance platform. Significance code: $* * * P<0.00 \mathrm{I}$.

Abbreviations: ADHD, Attention-deficit hyperactivity disorder; TD, typically developing.
Table I Performance data and between-group differences

\begin{tabular}{|c|c|c|c|c|c|c|c|}
\hline \multirow[t]{2}{*}{ Variables } & \multicolumn{2}{|c|}{$\begin{array}{l}\text { ADHD } \\
(n=62)\end{array}$} & \multicolumn{2}{|c|}{$\begin{array}{l}\text { TD } \\
(n=62)\end{array}$} & \multirow{2}{*}{$\frac{t \text {-test }}{P \text {-value }}$} & \multicolumn{2}{|c|}{ Effect size } \\
\hline & $M^{a}$ & SD & $M^{a}$ & SD & & $d$ & $r$ \\
\hline \multicolumn{8}{|l|}{ CPT-II v.5 } \\
\hline Omissions & 60.55 & 24.04 & 43.82 & 2.89 & 0.001 & 0.98 & 0.44 \\
\hline Comn & 47.93 & 9.97 & 51.19 & 9.26 & 0.06 & -0.34 & -0.17 \\
\hline Hit-RT & 56.85 & 11.05 & 42.01 & 8.78 & $<0.001$ & 1.49 & 0.60 \\
\hline Hit-SE & 58.37 & 9.43 & 40.54 & 5.53 & $<0.001$ & 2.30 & 0.76 \\
\hline Var-SE & 56.88 & 8.29 & 42.31 & 6.71 & & 1.93 & 0.69 \\
\hline DPrime & 51.10 & 9.23 & 51.64 & 8.15 & 0.73 & -0.06 & -0.03 \\
\hline Beta & 54.20 & 10.09 & 44.18 & 4.87 & $<0.001$ & 1.27 & 0.54 \\
\hline & 55.15 & 15.52 & 45.34 & 2.87 & & 0.88 & $0.4 I$ \\
\hline Hit-R & 52.27 & 9.64 & 46.25 & 6.82 & $<0.001$ & 0.72 & 0.34 \\
\hline Hit-S & 50.95 & 8.96 & 48.05 & 8.44 & & 0.33 & .17 \\
\hline Hit-F & 57.80 & 12.94 & 45.60 & 5.69 & $<0$ & 1.22 & 0.52 \\
\hline Hit-SE-ISI & 53.92 & 9.66 & 44.55 & 5.58 & $<0.001$ & 1.19 & 0.51 \\
\hline \multicolumn{8}{|c|}{ Cerebellar symptoms } \\
\hline ICARS & 3.17 & 1.28 & 1.44 & 1.28 & $<0.001$ & 0.84 & 0.39 \\
\hline \multicolumn{8}{|c|}{ Balance measures } \\
\hline Balance trial & $\mathbf{M}^{\mathrm{b}}$ & SD & $\mathbf{M}^{\mathrm{b}}$ & SD & $P$-value & $d$ & $\boldsymbol{r}$ \\
\hline LLI & 138.5 & 65.9 & 99.7 & 37.8 & $<0.001$ & 0.72 & 0.34 \\
\hline LL2 & 120.6 & 45.6 & 87.2 & 40.6 & $<0.00$ I & 0.77 & 0.36 \\
\hline LL3 & 127.5 & 48.6 & 82.4 & 38.4 & $<0.001$ & 1.03 & 0.46 \\
\hline LL4 & 122.5 & 43.1 & 76.0 & 36.7 & $<0.001$ & 1.16 & 0.51 \\
\hline LL5 & 127.3 & 42.7 & 78.7 & 33.0 & $<0.001$ & 1.28 & 0.54 \\
\hline \multicolumn{8}{|c|}{ Overall balance score } \\
\hline MeanBal & 4.9 & 0.6 & 4.3 & 0.6 & $<0.001$ & 1.17 & 0.51 \\
\hline
\end{tabular}

Notes: CPT-II T-scores: omissions, errors of omission; commissions, errors of commission; Hit-RT, mean reaction time; Hit-SE, standard error of reaction time; Var-SE, variability of standard error (ie, the variability of an individual's reaction time in relation to his overall standard error); DPrime, an individual's ability to discriminate between signal and noise; beta, response style, with higher beta values indicating an emphasis on avoiding commission errors; perseverations, responses that occur $<100 \mathrm{~ms}$ after a stimulus; Hit-RT-Block, the slope of the changes in reaction times over the 6 time blocks; Hit-SE-Block, the slope of the changes in the standard errors of the reaction times over the 6 time blocks; Hit-RT-ISI, the slope of the changes in reaction times over three interstimulus intervals (ISIs; I, 2, and 4 seconds); Hit-SE-ISI, the slope of the changes in the standard errors of the reaction times over three interstimulus intervals; ICARS, number of symptoms measured using the international scale for rating ataxias. Higher number means worse performance. MeanBal, overall balance score - the arithmetic average of the

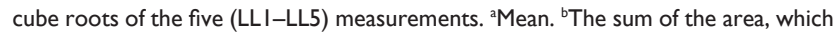
is defined by the path of a point oscillating from the imaginary center and a line bisecting the center.

Abbreviations: ADHD, attention-deficit hyperactivity disorder; CPT-II, Continuous Performance Test-II; ICARS, International Cooperative Ataxia Rating Scale; LL, laterolateral; SD, standard deviation; TD, typically developing.

No correlations were observed between overall balance score (MeanBal) and overall ADHD symptom score obtained from the parent form of CSI-4 or with the IA and HI subscales. A regression model for the analysis of the combined dataset that controlled for the influences of age and group revealed a tendency for the parent-reported HI score to increase as the overall balance performance worsened.

\section{Balance and CPT-II performances}

We observed that the overall balance score (MeanBal) in both groups exhibited relationships with the variables that 
described the variation in the reaction time on the CPT-II including Hit Reaction time Standard Error (RT-SE) $(r=0.25$; $P=0.0409$, respectively, $r=0.31 ; P=0.0131)$ and Variability of Standard Error (Var-SE) $(r=0.28 ; P=0.0252$, respectively, $r=0.41 ; P<0.001)$. Furthermore, in the TD group, overall balance score correlated with an increase in the reaction time over the six blocks of CPT stimuli (RT-Block) $(r=0.26$; $P=0.0423$ ) and with an increase in standard error of the reaction time over the course of these tasks (RT-SE-Block) $(r=0.45 ; P<0.001)$.

In the regression analyses that were performed separately for each group, we observed that an increase in the overall balance score led to increases in the variables RT-SE and Var-SE in both the children with ADHD and the TD children (Table 2).

Table 2 Age-corrected regression coefficients and $P$-values measuring the influence of the overall balance score (MeanBal) on ADHD symptom scores and performance in CPT-II

\begin{tabular}{|c|c|c|c|c|c|c|}
\hline \multicolumn{7}{|c|}{ Influence of worsening of overall balance performances } \\
\hline \multirow[t]{2}{*}{ Variables } & \multicolumn{2}{|c|}{ ADHD $(n=62)$} & \multicolumn{2}{|c|}{ TD $(n=62)$} & \multicolumn{2}{|c|}{ Total $(n=124)$} \\
\hline & $\overline{\mathrm{Cft}}$ & $P$-value & $\overline{\mathrm{Cft}}$ & $P$-value & $\overline{\mathrm{Cft}}$ & $P$-value \\
\hline \multicolumn{7}{|c|}{ CSI-4 (T-score) } \\
\hline ADHD total & -0.64 & 0.8302 & 5.07 & 0.0852 & 2.25 & 0.2805 \\
\hline IA & 0.96 & 0.7474 & 2.97 & 0.2520 & 2.04 & 0.2957 \\
\hline $\mathrm{HI}$ & 2.43 & 0.3803 & 5.71 & 0.0725 & 3.99 & 0.0555 \\
\hline ODD & 1.13 & 0.6810 & -2.08 & 0.3048 & -0.61 & 0.7197 \\
\hline \multicolumn{7}{|c|}{ CPT-II (T-score) } \\
\hline Omissions & 5.33 & 0.3503 & 0.53 & 0.4496 & 2.78 & 0.3349 \\
\hline Commissions & 3.71 & 0.1181 & 0.10 & 0.9644 & 1.84 & 0.2563 \\
\hline RT & 1.03 & 0.6972 & 2.37 & 0.2483 & 1.75 & 0.2929 \\
\hline RT-SE & 3.97 & 0.0431 & 2.66 & 0.0220 & 3.37 & 0.0067 \\
\hline Var-SE & 4.07 & 0.0372 & 3.97 & 0.0049 & 4.13 & 0.0006 \\
\hline DPrime & 1.83 & 0.4098 & -2.10 & 0.2830 & -0.20 & 0.8901 \\
\hline Beta & 0.83 & 0.7306 & -0.24 & 0.8425 & 0.26 & 0.8474 \\
\hline Perseverations & 2.34 & 0.5246 & 1.22 & 0.0760 & 1.64 & 0.3795 \\
\hline RT-Block & 1.16 & 0.6151 & 4.15 & 0.0105 & 2.70 & 0.0540 \\
\hline RT-SE-Block & 2.35 & 0.2614 & 7.50 & $<0.001$ & 4.59 & 0.0015 \\
\hline RT-ISI & 0.62 & 0.8410 & -0.36 & 0.7964 & 0.12 & 0.9433 \\
\hline RT-SE-ISI & 2.57 & 0.2649 & 0.80 & 0.5516 & 1.71 & 0.1955 \\
\hline
\end{tabular}

Notes: In the combined sample, the effects of the overall balance score (MeanBal) is corrected also for group (ADHD/TD). CSI-4 - Parent version: ADHD total, T-score of ADHD scale of the CSI-4; IA, inattentive subscale of the CSI-4; HI, hyperactiveimpulsive subscale of the CSI-4; CPT-II T-scores: omissions, errors of omission; commissions, errors of commission; RT, mean reaction time; RT-SE, standard error of reaction time; Var-SE, variability of standard error (ie, the variability of an individual's reaction time in relation to his overall standard error); D-prime, an individual's ability to discriminate between signal and noise; beta, response style, with higher beta values indicating an emphasis on avoiding commission errors; perseverations, responses that occur $<100 \mathrm{~ms}$ after a stimulus; RT-Block, the slope of the changes in reaction times over the 6 time blocks; RT-SE-Block, the slope of the changes in the standard errors of the reaction times over the 6 time blocks; RT-ISI, the slope of the changes in reaction times over three interstimulus intervals (ISIs; I, 2, and 4 seconds); RT-SE-ISI, the slope of the changes in the standard errors of the reaction times over three ISI.

Abbreviations: ADHD, attention-deficit hyperactivity disorder; CPT-II, Continuous Performance Test-II; CSI-4, Child Symptom Inventory-4; ICARS, International Cooperative Ataxia Rating Scale; LL, laterolateral; SD, standard deviation; TD, typically developing; ODD, oppositional defiant disorder; Cft, coefficient.

\section{Relationship between the balance performance and the cerebellar symptom score (ICARS)}

We found that the cerebellar symptom score according to the ICARS was strongly related to the overall balance performance (MeanBal) in both groups (ADHD: $r=0.50$, $P<0.001$; TD: $r=0.49, P=0.001$ ).

\section{Discussion}

In the current study, we have shown that the ADHD group exhibited a considerably worse ability to maintain or improve dynamic balance compared to the TD children. Our results correspond with a finding from Mao et a ${ }^{11}$ who observed an impairment in dynamic balance in children with ADHD and with a German study in which the impairment in dynamic posturography observed in children with ADHD was similar to that observed in children with cerebellar lesions. ${ }^{6}$

We did not find a relationship between score of ADHD symptoms and balance performance. Similarly, Ferrin and Vance, ${ }^{4}$ in a much larger sample of children and adolescents with ADHD than ours, established only weak correlations between neurological soft signs and ADHD symptoms.

We observed relationships between balance performance and several variables of the CPT-II. The most consistent were the relationships of the overall balance score (MeanBal) with the variables that described the reaction time variability (ie, Hit-RT-SE and Var-SE). Variability in reaction times belongs to one of the most consistent neuropsychological findings in individuals with ADHD. ${ }^{31}$ However, it may reflect various underlying cognitive processes.

RT-SE reflects the extent to which longer reaction times occur in an otherwise normal or rapid performance..$^{37,38}$ According to current hypotheses, the neurobiological basis of RT-SE is the reduced ability to suppress the task-negative default mode network, due to differences in the connectivity of the dorsolateral prefrontal cortex and the cerebellum..$^{39,40}$ Ghajar and Ivry ${ }^{41}$ interpret the role of the cerebellum in attention through its ability to create predictive settings in the CNS. Thus, the relationship between balance performance and the RT-SE variable could reflect damage to predictive settings in both the motor and the cognitive areas. This line of thinking is supported by results of our previous study in children with ADHD in which we found that higher score of cerebellar symptoms predicted an increase in RT-SE. ${ }^{10}$

We found a strong correlation between overall balance performance and cerebellar symptoms. This finding is consistent with the fact that the cerebellum is the central 
structure in the regulation of balance. ${ }^{16}$ Because of its enormous computational capacity, the cerebellum enables the integration of information from a variety of modalities and also enables the modeling of the future state of an object, which is essential for proactive anticipatory control. ${ }^{42,43}$ This process is critical for dynamic balance. Structural abnormalities in the cerebellum are consistently found in imaging studies of ADHD and are related to the overall volumes ${ }^{18}$ of the hemispheres. ${ }^{44}$ However, findings related to the vermis area predominate. ${ }^{17,19-21,45-47}$ The vermis is a centrally located part of the paleocerebellum that participates in the regulation of muscle tension and balance. The posterior-inferior area of the vermis has recently been shown to be activated during cognitive-emotional tasks, ${ }^{48}$ which would explain the fact that the presence of structural abnormalities in vermis are not solely associated with motor deficiencies but also with clinical prognosis of ADHD. ${ }^{47}$

The variables reflecting inconsistency of reactions (the CPT-II Var-SE) was also found to be related to balance performance in our study. Var-SE characterizes the overall variety of the reaction times and reflects the true diversity of the reaction times in the test. ${ }^{49}$ It has been suggested that this type of inconsistency indicates a fault in sensorimotor regulation. ${ }^{50}$ In the brain, sensorimotor regulation is related to regions including the presupplementary motor area (PreSMA), the supplementary motor (SMA) cortex, the primary motor cortex, the basal ganglia, and the cerebellum. ${ }^{51}$ The PreSMA and SMA receive direct input from the basal ganglia and the cerebellum. ${ }^{52}$ Differentiation of the neural substrate of go phase and no-go phase tests revealed that the go phase in the CPT is related to the activation of the left primary sensorimotor cortex, the SMA, and the cerebellum. ${ }^{53}$ In TD children, lower variations in reaction time have been linked to the activations of the PreSMA and SMA, whereas children with ADHD must compensate for dysfunctional premotor regulation with an increase in the activation of the prefrontal cortex.${ }^{54}$ Cerebellar dysfunction would then contribute to the intrasubjective variability of reaction time precisely by interrupting automatic premotor regulation. This may increase demands on prefrontal control, which may then contribute to worse performance in other cognitive functioning due to the lack of capacity and fatigue.

\section{Limitations}

Several limitations can be kept in mind when considering the results of this study. First, the evaluation of cerebellar symptoms was not conducted blind to ADHD vs TD group status. Evaluation of actual balance was conducted via computerized measurement, but the individual supervising the assessment was not blind to group status. We also did not use a direct cerebellar measure, we only indirectly measured clinical cerebellar symptoms. It is noteworthy that about half of the children with ADHD suffered from comorbid ODD. Neurologic soft signs have also been found in children with externalizing disorders. ${ }^{4}$ Our sample also contained only few girls (seven), and therefore, we were not able to test whether balance performance is sex dependent. It has been found that girls and boys may differ in manifestation of $\mathrm{ADHD}^{55,56}$ and in motor deficiencies. ${ }^{56,57}$

We also cannot exclude that our study lacked power to detect a correlation between balance control and ADHD symptoms. The generalizability of our findings is also impacted by variance in methods of diagnosis of ADHD. It should be noted that different methods, in different settings, are likely to reveal somewhat different versions of ADHD. ${ }^{58}$ We also cannot exclude that our methods were inadequate to identify a common neurocognitive condition other than ADHD. ADHD is a considerably heterogeneous syndrome, and individuals with ADHD differ in their pattern of presenting symptoms. Our sample was not evenly distributed among ADHD subtypes, limiting our ability to make comparisons between these groups. Five percent of our sample of individuals with ADHD had prior methylphenidate treatment, so it is possible this group reflect a different kind or level of pathology than those that entered the study naive to medicine. However, our method of ascertainment of subjects means that our ADHD sample should be representative of other clinical samples. Comorbid neuropsychological and motor dysfunction occurs in $30 \%-50 \%$ of individuals with ADHD. ${ }^{1}$ We believe that our methods should have been sufficient to exclude neuropsychiatric syndromes identified by a trained specialist, which have a major cognitive or motor control component.

Finally, the study is cross-sectional; therefore, it limits us making conclusion on the nature of the relationship between measured variables.

\section{Conclusion}

The present study showed that children with ADHD have worse dynamic balance performance. Clinicians should therefore be sensitive to the possibility that balance performance is an important clinical symptom in ADHD children. We also described a relationship between balance and cognitive processes involved in control of reactions. Assessment techniques that systematically identify impaired reaction 
and balance and clinical interventions that support improved control over reaction time or cerebellar function may reduce morbidity related to balance impairment.

\section{Acknowledgments}

This study was supported by Charles University Grant GAUK 383/10, the Program for the Development of Fields of Study at Charles University (P07 Psychosocial Aspects of the Quality of Human Life), and GACR (The Czech Science Foundation) 16-2179S.

\section{Disclosure}

Dr Goetz serves as a speaker for Eli Lilly CR. Dr Surman reports that since the last year, he has been a consultant to Ironshore, Rhodes, and Shire; has presented educational lectures for Neos, Graduate Medical Education Continuing Medical Education (CME), and Massachusetts General Hospital (MGH) Academy; and has received research support from Neurocentria, Pamlab, and Shire. He also reports receiving royalties for Fast Minds: How to Thrive If You Have ADHD (Or Think You Might) and ADHD in Adults: A Practical Guide to Evaluation and Management. Dr Schwabova, Dr Hlavka, and Dr Ptacek report no conflicts of interest in this work.

\section{References}

1. Landgren MM, Pettersson RR, Kjellman BB, Gillberg CC. ADHD, DAMP and other neurodevelopmental/psychiatric disorders in 6-year-old children: epidemiology and co-morbidity. Dev Med Child Neurol. 1996; 38(10):891-906.

2. Udal AH, Malt UF, Lövdahl H, Gjaerum B, Pripp AH, Groholt B. Motor function may differentiate attention deficit hyperactivity disorder from early onset bipolar disorder. Behav Brain Funct. 2009;5(1):47.

3. Gustafsson P, Svedin CG, Ericsson I, Lindén C, Karlsson MK, Thernlund G. Reliability and validity of the assessment of neurological soft-signs in children with and without attention-deficit-hyperactivity disorder. Dev Med Child Neurol. 2010;52(4):364-370.

4. Ferrin M, Vance A. Examination of neurological subtle signs in ADHD as a clinical tool for the diagnosis and their relationship to spatial working memory. J Child Psychol Psychiatry. 2011;53(4):390-400.

5. Konicarova J, Bob P, Raboch J. Persisting primitive reflexes in medication-naïve girls with attention-deficit and hyperactivity disorder. Neuropsychiatr Dis Treat. 2013;9:1457-1461.

6. Buderath P, Gärtner K, Frings M, et al. Postural and gait performance in children with attention deficit/hyperactivity disorder. Gait Posture. 2009;29(2):249-254.

7. Konicarova J, Bob P, Raboch J. Balance deficits and ADHD symptoms in medication-naïve school-aged boys. Neuropsychiatr Dis Treat. 2014; 10:85-88.

8. Jacobi-Polishook T, Shorer Z, Melzer I. The effect of methylphenidate on postural stability under single and dual task conditions in children with attention deficit hyperactivity disorder - a double blind randomized control trial. J Neurol Sci. 2009;280(1-2):15-21.

9. Ghanizadeh A. Predictors of postural stability in children with ADHD. J Atten Disord. 2011;15(7):604-610.

10. Goetz M, Schwabova J, Hlavka Z, et al. Cerebellar symptoms are associated with omission errors and variability of response time in children with ADHD. J Atten Disord. 2017;21(3):190-199.
11. Mao H-Y, Kuo L-C, Yang A-L, Su C-T. Research in developmental disabilities balance in children with attention deficit hyperactivity disorder-combined type. Res Dev Disabil. 2014;35(6):1252-1258.

12. Hove MJ, Zeffiro TA, Biederman J, Li Z, Schmahmann J, Valera EM. Postural sway and regional cerebellar volume in adults with attentiondeficit/hyperactivity disorder. Neuroimage Clin. 2015;8(C):422-428.

13. Shilon Y, Pollak Y, Aran A, Shaked S, Gross-Tsur V. Accidental injuries are more common in children with attention deficit hyperactivity disorder compared with their non-affected siblings. Child Care Health Dev. 2012;38(3):366-370.

14. Kang J-H, Lin H-C, Chung S-D. Attention-deficit/hyperactivity disorder increased the risk of injury: a population-based follow-up study. Acta Paediatr. 2013;102(6):640-643.

15. Ren Y, Yu L, Yang L, Cheng J, Feng L, Wang Y. Postural control and sensory information integration abilities of boys with two subtypes of attention deficit hyperactivity disorder: a case-control study. Chin Med J. 2014;127(24):4197-4203.

16. Winter DA, Patla AE, Frank JS. Assessment of balance control in humans. Med Prog Technol. 1990;16(1-2):31-51.

17. Mostofsky SH, Reiss AL, Lockhart P, Denckla MB. Evaluation of cerebellar size in attention-deficit hyperactivity disorder. J Child Neurol. 1998;13(9):434-439.

18. Castellanos FX, Lee PP, Sharp W, et al. Developmental trajectories of brain volume abnormalities in children and adolescents with attentiondeficit/hyperactivity disorder. JAMA. 2002;288(14):1740-1748.

19. Bussing R, Grudnik J, Mason D, Wasiak M, Leonard C. ADHD and conduct disorder: an MRI study in a community sample. World J Biol Psychiatry. 2002;3(4):216-220.

20. Hill DE, Yeo RA, Campbell RA, Hart B, Vigil J, Brooks W. Magnetic resonance imaging correlates of attention-deficit/hyperactivity disorder in children. Neuropsychology. 2003;17(3):496-506.

21. Bledsoe J, Semrud-Clikeman M, Pliszka SR. A magnetic resonance imaging study of the cerebellar vermis in chronically treated and treatment-naïve children with attention-deficit/hyperactivity disorder combined type. Biol Psychiatry. 2009;65(7):620-624.

22. Bledsoe JC, Semrud-Clikeman M, Pliszka SR. Neuroanatomical and neuropsychological correlates of the cerebellum in children with attention-deficit/hyperactivity disorder-combined type. J Am Acad Child Adolesc Psychiatry. 2011;50(6):593-601.

23. Koziol LF, Budding D, Andreasen N, et al. Consensus paper: the cerebellum's role in movement and cognition. Cerebellum. 2014;13(1): 151-177.

24. Ogg RJ, Zou P, Allen DN, Hutchins SB, Dutkiewicz RM, Mulhern RK. Neural correlates of a clinical continuous performance test. Magn Reson Imaging. 2008;26(4):504-512.

25. Tana MG, Montin E, Cerutti S, Bianchi AM. Exploring cortical attentional system by using fMRI during a Continuous Perfomance Test. Comput Intell Neurosci. 2010.

26. Gadow KD, Sprafkin JN. Child Symptom Inventory 4: Screening and Norms Manual. Stony Brook, NY: Checkmate Plus; 2002:179.

27. Ondrejka I, Abali O, Paclt I, et al. A prospective observational study of attention-deficit/hyperactivity disorder in Central and Eastern Europe and Turkey: symptom severity and treatment options in a paediatric population. Int J Psychiatry Clin Pract. 2010;14(2):116-126.

28. Goetz M, Yeh CB, Ondrejka I, et al. A 12-month prospective, observational study of treatment regimen and quality of life associated with ADHD in Central and Eastern Europe and eastern Asia. J Atten Disord. 2012;16(1): 44-59.

29. American Psychological Association. Diagnostic and Statistical Manual of Mental Disorders. 4th ed. Washington, DC; 1994.

30. Zang Y, Gu B, Qian Q, Wang Y. Objective measurement of the balance dysfunction in attention deficit hyperactivity disorder children. Chin J Clin Rehabil. 2002;6:1372-1374.

31. Trouillas $\mathrm{P}$, Takayanagi $\mathrm{T}$, Hallett $\mathrm{M}$, et al. International Cooperative Ataxia Rating Scale for pharmacological assessment of the cerebellar syndrome. The Ataxia Neuropharmacology Committee of the World Federation of Neurology. J Neurol Sci. 1997;145(2):205-211. 
32. Schmitz-Hübsch T, Tezenas du Montcel S, Baliko L, et al. Reliability and validity of the International Cooperative Ataxia Rating Scale: a study in 156 spinocerebellar ataxia patients. Mov Disord. 2006;21(5):699-704.

33. Storey E, Tuck K, Hester R, et al. Inter-rater reliability of the International Cooperative Ataxia Rating Scale (ICARS). Mov Disord. 2004;19(2): 190-192.

34. Frings M, Gaertner K, Buderath P, et al. Megalographia in children with cerebellar lesions and in children with attention-deficit/hyperactivity disorder. Cerebellum. 2010;9(3):429-432.

35. Conners CK. Conners' Continuous Performance Test (CPT II): Version 5 for Windows: Technical Guide and SoftWare Manual. Toronto, ON: Multi Health Systems; 2004.

36. R Development Core Team. R: A language and environment for statistical computing. Vienna, Austria: R Foundation for Statistical Computing; 2013.

37. Castellanos FX, Tannock R. Neuroscience of attention deficit/hyperactivity disorder: the search for endophenotypes. Nat Rev Neurosci. 2002;3(8): 617-628.

38. Leth-Steensen C, Elbaz ZK, Douglas VI. Mean response times, variability, and skew in the responding of ADHD children: a response time distributional approach. Acta Psychol (Amst). 2000;104(2):167-190.

39. Sonuga-Barke EJS, Castellanos FX. Spontaneous attentional fluctuations in impaired states and pathological conditions: a neurobiological hypothesis. Neurosci Biobehav Rev. 2007;31(7):977-986.

40. Fair DA, Nigg JT, Iyer S, et al. Distinct neural signatures detected for ADHD subtypes after controlling for micro-movements in resting state functional connectivity MRI data. Front Syst Neurosci. 2013;4(6):80.

41. Ghajar J, Ivry RB. The predictive brain state: asynchrony in disorders of attention? Neuroscientist. 2009;15(3):232-242.

42. Wolpert DM, Miall RC, Kawato M. Internal models in the cerebellum. Trends Cogn Sci. 1998;2(9):338-347.

43. Imamizu $H$, Kawato $M$. Brain mechanisms for predictive control by switching internal models: implications for higher-order cognitive functions. Psychol Res. 2009;73(4):527-544.

44. Ivanov I, Murrough JW, Bansal R, Hao X, Peterson BS. Cerebellar morphology and the effects of stimulant medications in youths with attention deficit-hyperactivity disorder. Neuropsychopharmacology. 2013;39(3): 718-726.

45. Berquin PC, Giedd JN, Jacobsen LK, et al. Cerebellum in attention-deficit hyperactivity disorder: a morphometric MRI study. Neurology. 1998; 50(4):1087-1093.
46. Castellanos FXF, Giedd JNJ, Berquin PCP, et al. Quantitative brain magnetic resonance imaging in girls with attention-deficit/hyperactivity disorder. Arch Gen Psychiatry. 2001;58(3):289-295.

47. Mackie SS, Shaw PW, Lenroot RR, et al. Cerebellar development and clinical outcome in attention deficit hyperactivity disorder. Am J Psychiatry. 2007;164(4):647-655.

48. Stoodley CJ, Schmahmann JD. Functional topography in the human cerebellum: a meta-analysis of neuroimaging studies. Neuroimage. 2009; 44(2):489-501.

49. Karalunas SL, Huang-Pollock CL, Nigg JT. Decomposing attentiondeficit/hyperactivity disorder (ADHD)-related effects in response speed and variability. Neuropsychology. 2012;26(6):684-694.

50. Manto M, Bower JM, Conforto AB, et al. Consensus paper: roles of the cerebellum in motor control - the diversity of ideas on cerebellar involvement in movement. Cerebellum. 2011;11(2):457-487.

51. Hart H, Radua J, Mataix-Cols D, Rubia K. Meta-analysis of fMRI studies of timing in attention-deficit hyper-activity disorder (ADHD). Neurosci Biobehav Rev. 2012;36(10):2248-2256.

52. Akkal D, Dum RP, Strick PL. Supplementary motor area and presupplementary motor area: targets of basal ganglia and cerebellar output. J Neurosci. 2007;27(40):10659-10673.

53. Mostofsky SH, Schafer JG, Abrams MT, et al. fMRI evidence that the neural basis of response inhibition is task-dependent. Brain Res Cogn Brain Res. 2003;17(2):419-430.

54. Suskauer SJ, Simmonds DJ, Caffo BS, Denckla MB, Pekar JJ, Mostofsky SH. fMRI of intrasubject variability in ADHD: anomalous premotor activity with prefrontal compensation. J Am Acad Child Adolesc Psychiatry. 2008;47(10):1141-1150.

55. Rucklidge JJ. Gender differences in attention-deficit/hyperactivity disorder. Psychiatr Clin North Am. 2010;33(2):357-373.

56. Ghezzo A, Guerini FR, Bolognesi E, et al. Neuropsycological gender differences in healthy individuals and in pediatric neurodevelopmental disorders. A role for SNAP-25. Med Hypotheses. 2009;73(6):978-980.

57. Chiappedi M, Togni R, De Bernardi E, et al. Arm trajectories and writing strategy in healthy children. BMC Pediatr. 2012;7(12):173.

58. Bonati M, Reale L, Zanetti M, et al; Lombardy ADHD Group. A regional ADHD center-based network project for the diagnosis and treatment of children and adolescents with ADHD. J Atten Disord. Epub 2015 Aug 28. pii: 1087054715599573.
Neuropsychiatric Disease and Treatment

\section{Publish your work in this journal}

Neuropsychiatric Disease and Treatment is an international, peerreviewed journal of clinical therapeutics and pharmacology focusing on concise rapid reporting of clinical or pre-clinical studies on a range of neuropsychiatric and neurological disorders. This journal is indexed on PubMed Central, the 'PsycINFO' database and CAS,

\section{Dovepress}

and is the official journal of The International Neuropsychiatric Association (INA). The manuscript management system is completely online and includes a very quick and fair peer-review system, which is all easy to use. Visit http://www.dovepress.com/testimonials.php to read real quotes from published authors. 